\title{
Pregnancy and its role in breast cancer
}

\author{
Filipe Correia Martins · Maria Filomena Botelho • \\ Mafalda Laranjo · António Manuel Cabrita · \\ Maria Isabel Torgal · Carlos Freire de Oliveira
}

Received: 21 July 2008 / Accepted: 2 August 2008 / Published online: 17 September 2008

CS Springer-Verlag 2008

\begin{abstract}
Early full-term pregnancy is the only recognized factor able to prevent breast cancer. There are several hypotheses to explain the mechanisms of this protection, namely an altered hormonal milieu, a differentiation process or a switch in stem cell properties. To explore them, authors have been using animal models, mainly in rodents. Hormonal administration with estrogen and progesterone was the most widely used process to mimic the mammary changes during pregnancy. We have recently
\end{abstract}

F.C. Martins ( $ه)$

Dept. of Gynecology, University Hospital of Coimbra, Portugal;

Biophysics and Biomathematics Institute, IBILI,

Faculty of Medicine of the University of Coimbra, Portugal;

CIMAGO (Oncology, Genetics and Environment Research Center),

Faculty of Medicine of the University of Coimbra, Portugal

e-mail: filipecorreiamartins@gmail.com

M.F. Botelho $\bullet$ M. Laranjo

Biophysics and Biomathematics Institute, IBILI,

Faculty of Medicine of the University of Coimbra, Portugal;

CIMAGO (Oncology, Genetics and Environment Research Center),

Faculty of Medicine of the University of Coimbra, Portugal

\section{A.M. Cabrita}

Experimental Pathology Institute,

Faculty of Medicine of the University of Coimbra, Portugal;

CIMAGO (Oncology, Genetics and Environment Research Center),

Faculty of Medicine of the University of Coimbra, Portugal

M.I. Torgal • C.F. de Oliveira

Dept. of Gynecology, University Hospital of Coimbra, Portugal;

CIMAGO (Oncology, Genetics and Environment Research Center),

Faculty of Medicine of the University of Coimbra, Portugal proposed that this enigmatic protective role of a full-term birth in breast cancer is carried out by tumor inhibition mediated by differentiated mammary epithelial cells. This explanation may give a new perspective of breast cancer prevention and treatment.

Keywords Pregnancy · Breast Cancer · Hormones · Mammary Gland · Carcinogenesis

\section{Introduction}

There are several established epidemiological factors that may influence breast cancer incidence, such as age, genetics, reproductive history (including parity, menarche, menopause and first pregnancy age and extent of lactation), family history of malignancies, personal history of breast disorders, place of residence, socioeconomic status, ethnicity or radiation $[1,2]$. Despite this variety, the only factor that may reduce the lifetime breast cancer risk is an early full-term pregnancy [3-5]. Although the exact mechanisms remain unknown, there are various theories and experimental models that try to clarify breast refractoriness to carcinogenesis after early pregnancy. This review, aims to discuss them, starting from an epidemiological approach and reflecting on future perspectives in this field.

\section{Epidemiology}

Women who have a child before 20 years of age have a significant decrease of about 50\% in the risk of developing breast tumors. Nevertheless, this risk is increased if they are older when they have a first full-term birth, when com- 
pared with the nulliparous [6]. Recent studies reveal that longer intervals between age of menarche and age of first birth are associated with an increased risk of hormonally sensitive types of breast cancer [7].

This protective role of pregnancy is not immediate and a transient increase after delivery was demonstrated.

Recent meta-analysis has demonstrated that there is a hormonal specificity in this pregnancy protection. Only hormone responsive breast tumors, which express estrogen receptors (ER) and progesterone receptors (PR), are susceptible to the beneficial effects of parity. Surprisingly, although most of the studies state no parity effect in negative ER and PR tumors, some reveal an increased risk of developing this molecular type of breast cancer after pregnancy [6]. Futhermore, the prolonged use of oral contraceptives may increase the ER rate within the tumor tissue; these can, therefore, be considered an indirect prognostic factor. [8]

The initial differentiation of epithelial cells during early pregnancy is not sufficient to protect against breast cancer. Although most of the studies demonstrate that aborted pregnancies are not associated with a different risk for breast cancer [9], some data show that there may be an increased risk among women who have undergone a termination [6]. Otherwise, the main protective influence of parity in breast cancer is the timing of the first full-term pregnancy rather than its occurrence per se. The association is independent of the number of live births [10].

Some epidemiological studies have shown that prolonged breast-feeding offers some slight protection against breast cancer risk [11].

\section{Mechanisms for breast cancer protection}

During a full-term pregnancy, the mammary gland undergoes a maturation step in which cellular differentiation occurs. After the delivery and lactation, in spite of the gland regression, there are still some features of differentiation which remain. The same occurrence is observed not only in humans but also in rodents $[12,13]$.

Different theories have been developed to explain the protective role of pregnancy. However, there are four main schools of thought which enjoy some consensus and have common aspects.

The first theory places the most important role of this parity protection in the hormonal changes, namely in estradiol, prolactin and growth hormone levels $[14,15]$. The second is based on the fact that pregnancy is associated with a cellular differentiation which is maintained after involution. It considers epithelial cells to have less ability to proliferate and then become less susceptible to carcino- genic stimulus [12]. The third hypothesis accepts the cancer stem cell theory [16]. It states that the protective role of pregnancy in breast cancer is due to the decreased number of mammary stem cells, with less epithelial precursors which are potentially susceptible to malignant transformation [17]. Finally, once the protection is specific for the positive ER tumors, it can be mediated by changes in the estrogen responsiveness, either directly or paracrinally, through ER dependent or independent mechanisms [6].

\section{Altered hormonal milieu}

One of the hypotheses is that the protective role of the pregnancy is related to permanent changes in the hormonal environment $[18,19]$. Both prolactin and growth hormone $(\mathrm{GH})$ circulating levels are reduced in parous when compared to the aged-matched virgin (AMV) animals [19, 20]. According to some authors, this decrease reduces the susceptibility to carcinogenic transformation by reducing the levels of ER and epithelial growth factor receptors (EGF-R) in the parous mammary gland [18].

\section{Induction of differentiation}

Some authors suggest that pregnancy and hormonal administration induces the differentiation of the mammary gland, inhibiting cancer initiation because of the removal of cancer-susceptible cells [21-23]. Carcinogen agents could only interact with the undifferentiated and highly proliferating mammary epithelium cells of the terminal ducts, which are transformed to less mitotic alveolar cells after a hormonal stimulation. Although this theory is very attractive, it does not explain why placental lactogen and perphenazine (a dopamine receptor inhibitor which releases prolactin) do not protect against breast cancer, despite promoting differentiation [11]. Furthermore, the parous involuted gland has the same degree of differentiation as the AMV gland. Thus, this hypothesis does not clearly explain the difference in their behavior when submitted to carcinogenic exposure.

\section{Cell fate hypothesis}

According to the cell fate hypothesis, during adolescence, the hormonal environment promotes a molecular switch in stem cells, making them resistant to any carcinogenic stimulus through persistent differences in signal transduction pathways. Breast cancer would originate in undifferentiated terminal structures of the mammary gland (lobules type 1) that would contain stem cells 1 , the target of the neoplastic event. Early parity would produce a distinct type of cells, stem cells 2 , by a differentiation process of the mam- 
mary gland [24]. Despite differentiation, the mammary epithelium remains capable of responding with proliferation to a given stimuli, such as a new pregnancy. Under these circumstances, however, the cells that are stimulated to proliferate would be those in structures that had already been primed by the first cycle of differentiation. Therefore, they are able to metabolize the carcinogen and repair the induced DNA damage more efficiently than the cells of the nulliparous gland, being then less susceptible to carcinogenesis. However, if the shifting of stem cells 1 to stem cells 2 has not been completed, a powerful enough carcinogenic stimulus may overburden the system, thereby successfully initiating a neoplastic process. This hypothesis would explain the pregnancy blockage in carcinogeninduced proliferation [25].

\section{Experimental models}

The rat has been used as an ideal model for mechanistic studies about human breast cancer, namely to investigate the protective role of pregnancy. First of all, we have much information about the physiological role of hormones in the development and carcinogenesis of the rat mammary gland. Afterwards, in this animal species, parity prevents most of the mammary tumors and this can be mimicked by hormonal administration. Finally, there are many similarities between rat and human mammary glands and adenocarcinomas [26]. Both radiation and various chemicals are potent carcinogens able to induce mammary tumors in female rats. The most commonly used carcinogens are 7, 12-dimethylbenzanthracene (DMBA) [26] and methylnitrosourea (MNU) [27]. Although there are slight differences within strains, mammary tumors can be induced in several inbred or outbred rat strains, such as Sprague-Dawley (NSD), and Wistar-Furth (WF) rats. Female rats less than 55 days old produce two or three times more carcinomas than older rats 100 days old, demonstrating an age-related susceptibility to mammary carcinogenesis. After this, most of the mammary tumors arising at 50-55 days of age are ovarian-dependent carcinomas [26] and develop primarily from terminal end buds and terminal ducts [26, 28-30], although, in mature parous rats, mammary tumors are hormone-independent carcinomas and originate in the ducts. However, the carcinogenic mechanism and the progression of normal epithelium to intraductal hyperplasia/papilloma and carcinoma seem to be the same. Almost none of the experimental models of breast cancer present metastasis during the 3-6 months of the study's duration, with the exception of a few studies which reported metastases in rats splenectomized prior to carcinogen treatment $[28,31]$. There are several mechanisms that might prevent tumor formation, namely the suppression of carcinogenesis or the blocking of cancer promotion. The protection against breast cancer in experimental models can be achieved, for instance, by the administration of a combination of antioxidant micronutrients, avoiding those tumors with better prognosis $[32,33]$. Prevention of mammary carcinogenesis can also be obtained by a single pregnancy or by hormonal regimens comprising progesterone and estrogen or human chorionic gonadotropin [11]. Since the protective role of pregnancy can not be completely explained, there have been many experimental models trying to demonstrate parity-induced protection, either physiologically or by hormone administration. The two main classical models are the pre-treatment and posttreatment. In the first, there is an initial hormonal stimulation after which the gland involutes. The carcinogen is then administered to the hormone-treated and to the AMV animal of the control group. In the second animal model, rats are treated with the carcinogen and then exposed to the hormonal stimulus for a certain period. Pregnancy, both prior and following carcinogen exposure, reduces the incidence of mammary tumor and the number of carcinoma per rat, and significantly prolongs the latency period. There is a long-lasting protective effect of pregnancy, which was present even when the carcinogen was administered 100-130 days after the parturition [11]. Lactation following pregnancy does not seem to have any additive effect in breast cancer suppression, although a small sample size study gave opposite results [34]. The effect of interruption of pregnancy has also been studied in experimental models, but two different studies gave diverging results. One study shows that there is some small protection [35] while the other shows that a pregnancy interrupted at mid-term confers the same protection [36]. We consider the first work to have more powerful data as the animal groups involved were much larger.

\section{Mice models}

Although most of the experimental studies were performed in rats, the protective effect of pregnancy has also been demonstrated in mice. Initially, authors demonstrated that carcinogenic induction was inhibited in lactating mice [37]. It was later shown that involuted parous mammary gland was also unsusceptible to carcinogenic stimulus, although a comparison with an AMV mice group to exclude the possibility of the age-related refractoriness was not made [20]. More recently, this comparison was carried out in BD2fF1 and $\mathrm{C} 3 \mathrm{H} / \mathrm{Sm}$ mice, showing that mice are also a good model for studying pregnancy protection against breast cancer [38]. 


\section{Hormonal mimicry of pregnancy}

Pregnancy can be mimicked by hormonal treatment, either by using estrogen and progesterone or by using human chorionic gonadotropin [39], which is responsible for a rise in endogenous sex steroids. Both models were, therefore, used and were effective in reducing the incidence of mammary tumors in rodents [40]. Several studies demonstrated that, although there is a slight protection conferred by the separate use of estrogen or progesterone, the full protective effect is only achieved when both are used [11]. With regards to the doses and duration of the hormonal administration, we conclude that modest doses ( $20 \mu \mathrm{g}$ estrogen and $20 \mathrm{mg}$ progesterone) can be sufficient to prevent mammary carcinogenesis when administrated for 21 days in rats. However, if we use lower doses or the same doses for shorter periods of time, only partial protection is obtained [41]. There is, therefore, available evidence that the duration of estrogen and progesterone treatment, as well as the animal age, is an important factor in the protection against breast cancer [42]. Chorionic tropin also has a dosedependent effect when administered for 21 days, before the DMBA carcinogenic stimulus, with higher doses reducing the incidence and the number of tumors per rat [43]. The effect of estrogen and progesterone has been further studied in mice models with disrupted pathways [44]. As there is an overexpression of the HER2/neu oncogene in up to $30 \%$ of breast cancer, this hormonal protective effect was studied in mice overexpressing the referred gene (MMTVneu mice), revealing a reduction in the incidence of mammary tumors of more than $60 \%$. The p53 pathway is also disrupted in breast cancer and both p53 dependent and independent pathways are responsible for the hormonal protection. Apoptosis and cell arrest are acute responses mediated by p53 after carcinogenic stimulus, and increase following progesterone and estrogen treatment [45]. Nevertheless, some authors demonstrated that p53 independent pathways were also activated by exogenous hormones, with a $70 \%$ reduction in the incidence of mammary tumor incidence, using p53-deficient mice [46]. This preventive effect seems to be restricted to tumor progression, while preneoplastic lesions are still developing. Even when the estrogen and progesterone treatment was given to older mice (23-25 weeks old) the protective effect of the hormonal administration was preserved.

\section{Future perspectives}

A recent in vitro study showed that normal mammary epithelial cells inhibit breast cancer cell growth. This process was mediated by maspin and insulin growth factor- binding protein (IGF-BP) [47]. We have developed an in vivo model which corroborates these results (unpublished data) showing that the protective role of pregnancy in breast cancer may be carried out by the increase in the number of differentiated epithelial cells which produce inhibitory factors for the surrounding environment. After carcinogenic stimulus in the mammary gland, we may find carcinogen initiated cells. However, the promotion and the progression are blocked by a previous pregnancy or by a hormonal treatment [48]. According to our results, this blockage is related to the inhibitory factors produced by the differentiated epithelial cells, which are highly developed in these circumstances. An understanding of these inhibitory pathways may clarify the protective role of pregnancy in breast cancer and also contribute to the development of prevention and treatment strategies in the near future.

Conflict of interest statement The author declare that they have no conflict of interest to the publication of this article.

\section{References}

1. Kelsey JL, Berkowitz GS (1988) Perspectives in Cancer Research. Breast Cancer Epidemiology. Cancer Res 48:5615-5623

2. McPherson K, Steel CM, Dixon JM (2000) ABC of breast diseases. Breast cancer-epidemiology, risk factors, and genetics. Br J Cancer 321:624-628

3. MacMahon B, Cole P, Lin TM et al (1970) Age at first birth and breast cancer risk. Bull World Health Organ 43:209-221

4. Henderson BE, Powell D, Rosario I et al (1974) An epidemiologic study of breast cancer. J Natl Cancer Inst 53:609-614

5. Kelsey JL, Gammon MD, John EM (1993) Reproductive factors and breast cancer. Epidemiol Rev 15:36-47

6. Britt K, Ashworth A, Smalley M (2007) Pregnancy and the risk of breast cancer. Endocr Relat Cancer14:907-933

7. Li CI, Malone KE and Daling JR (2008) Timing of menarche and first fullterm birth in relation to breast cancer risk. Am J Epidemiol 167:230-239

8. Lumachi F, Ermani M, Marino F et al (2008) Relationship between oral contraceptive therapy and estrogen receptor status in patients with breast cancer. Anticancer Res 28:491-493

9. Henderson KD, Sullivan-Halley J, Reynolds P et al (2008) Incomplete pregnancy is not associated with breast cancer risk: the California Teachers Study. Contraception 77:391-396

10. Henderson BE, Ross RK and Pike MC (1991) Toward the primary prevention of cancer. Science 254:1131-1138

11. Sivaraman L and Medina D (2002) Hormone-Induced Protection Against Breast Cancer. J Mammary Gland Biol Neoplasia 7:77-92

12. Russo J, Tay LK and Russo IH (1982) Differentiation of the mammary gland and susceptibility to carcinogenesis. Breast Cancer Res Treat 2:5-73

13. Cardiff RD and Wellings SR (1999) The comparative pathology of human and mouse mammary glands. J Mammary Gland Biol Neoplasia 4:105-122

14. Emerman JT, Leahy M, Gout PW, Bruchovsky N (1985) Elevated growth hormone levels in sera from breast cancer patients. Horm Metab Res 17:421-424

15. Henderson BE, Feigelson HS (2000) Hormonal carcinogenesis. Carcinogenesis 21:427-433 
16. Trosko JE, Chang CC (1989) Stem cell theory of carcinogenesis. Toxicol Lett 49:283-295

17. Smalley M, Ashworth A (2003) Stem cells and breast cancer: a field in transit. Nat Rev Cancer 3:832-844

18. Welsch CW, Nagasawa H (1977) Prolactin and murine mammary tumorigenesis: A review. Cancer Res 37:951-963

19. Musey VC, Collins DC, Musey PI, Martino-Saltzman D and Preedy JR (1987). Long-term effect of a first pregnancy on the secretion of prolactin. N Engl J Med 316:229-234

20. Swanson SM, Guzman RC, Collins G et al (1995) Refractoriness to mammary carcinogenesis in the parous mouse is reversible by hormonal stimulation induced by pituitary isografts. Cancer Lett 90:171-181

21. Russo J and Russo IH (1997) Role of differentiation in the pathogenesis and prevention of breast cancer. Endocr Rel Cancer $4: 7-12$

22. Thordarson G, Jin E, Guzman RC et al (1995) Refractoriness to mammary tumorigenesis in parous rats: is it caused by persistent changes in the hormonal environment or permanent biochemical alterations in the mammary epithelia? Carcinogenesis $16: 2847-2853$

23. Abrams TA, Guzman RC, Hirokawa Y et al (1997) Refractoriness of parous rats to mammary carcinogenesis is overcome by treatment with ovarian hormones. Proc Am Assoc Cancer Res 38:1992

24. Russo J, Balogh G, Mailo Det al (2007) The genomic signature of breast cancer prevention. Recent Results Cancer Res 174:131-150

25. Russo J, Moral R, Balogh GA et al (2005) The protective role of pregnancy in breast cancer. Breast Cancer Res 7:131-142

26. Russo J, Gusterson BA, Rogers AE et al (1990) Comparative study of human and rat mammary tumorigenesis. Lab Invest 62:244-278

27. Gullino PM, Pettigrew HM, Grantham FH (1975) N-nitrosomethylurea as mammary gland carcinogen in rats. J Natl Cancer Inst 54:401-414

28. Fisher ER, Shoemaker RH, Sabnis A (1975) Relationship of hyperplasia to cancer in 3-methylcholanthrene-induced mammary tumorogenesis. Lab Invest 33:33-42

29. Haslam SZ, Bern HA (1977) Histopathogenesis of 7,12dimethyl benz(a)anthracene-induced rat mammary tumors. Proc Natl Acad Sci USA 74:4020-4024

30. Middleton PJ (1965) The histogenesis of mammary tumours induced in the rat by chemical carcinogens. $\mathrm{Br} \mathrm{J}$ Cancer $19: 830-839$

31. Kim U (1970) Metastasizing mammary carcinomas in rats: Induction and study of their immunogenicity. Science 167:72-74

32. Dias MF, Sousa E, Cabrita S et al (2000) Chemoprevention of DMBA-Induced Mammary Tumors in Rats by a Combined Regimen of Alpha-Tocopherol, Selenium, and Ascorbic Acid. Breast J 6:14-19
33. Martins FC, Teixeira F, Reis I, Geraldes N, Cabrita AM and Dias MF. Chemoprevention and Prognosis in Breast Cancer. Breast $\mathrm{J}$. in press

34. Yang J, Yoshizawa K, Nandi S et al (1999) Protective effects of pregnancy and lactation against $\mathrm{N}$-methyl- $\mathrm{N}$-nitrosoureainduced mammary carcinomas in female Lewis rats. Carcinogenesis 20:623-628

35. Sinha DK, Pazik JE, Dao TL (1988) Prevention of mammary carcinogenesis in rats by pregnancy: effect of full-term and interrupted pregnancy. Br J Cancer 57:390-394

36. Russo J, Rivera R, Russo IH (1992). Influence of age and parity on the development of the human breast. Breast Cancer Res Treat 23:211-218

37. Marchant J (1958) The inhibitory effect of continued lactation on the incidence of chemically induced breast tumors in mice of the IF strain. Br J Cancer 12:55-61

38. Medina D, Smith GH (1999) Chemical Carcinogen-Induced Tumorigenesis in Parous, Involuted Mouse Mammary Glands. J Natl Cancer Inst 91:967-969

39. Shi S, Xu L, Zhao G et al (2006) Apoptosis and tumor inhibition induced by human chorionic gonadotropin beta in mouse breast carcinoma. J Mol Med 84:933-941

40. Rajkumar L, Guzman RC, Yang J et al (2001) Short-term exposure to pregnancy levels of estrogen prevents mammary carcinogenesis. PNAS 98:11755-11759

41. Sivaraman L, Stephens LC, Markaverich BM (1998) Hormoneinduced refractoriness to mammary carcinogenesis in WistarFurth rats. Carcinogenesis 19:1573-1581

42. Tsubura A, Uehara N, Matsuoka Y et al (2008) Estrogen and progesterone treatment mimicking pregnancy for protection from breast cancer. In Vivo 22:191-201

43. Russo IH, Gimotty P, Dupuis M et al (1989) Effect of medroxyprogesterone acetate on the response of the rat mammary gland to carcinogenesis. Br J Cancer 59:210-216

44. Jerry DJ (2007) Roles for estrogen and progesterone in breast cancer prevention. Breast Cancer Res 9:102

45. Becker KA, Lu S, Dickinson ES et al (2005) Estrogen and progesterone regulate radiation-induced p53 activity in mammary epithelium through TGF-beta dependent pathways. Oncogene 24:6345-6353

46. Rajkumar L, Kittrell FS, Guzman RC et al (2007) Hormoneinduced protection of mammary tumorigenesis in genetically engineered mouse models. Breast Cancer Res 9:R12

47. Toillon R, Lagadec C, Page A et al (2007) Proteomics Demonstration That Normal Breast Epithelial Cells Can Induce Apoptosis of Breast Cancer Cells through Insulin-like Growth Factor-binding Protein-3 and Maspin. Mol Cell Proteomics 6:1239-1247

48 Lakshmanaswamy R, Guzman RC, Nandi S (2008) Hormonal prevention of breast cancer: significance of promotional environment. Adv Exp Med Biol 617:469-475 